\title{
Trends in dental caries experience of school children in Australia - 1977 to 1993
}

\author{
M. J. Davies* \\ A. J. Spencer ${ }^{\star}$ \\ G. D. Slade $\dagger$
}

\begin{abstract}
Data on the dental health of Australian school children from 1977 to 1985 have previously been reported. Significant features included a secular decline in caries experience as defined by the number of decayed, missing and filled teeth in both the deciduous dentition (dmft index) and permanent dentition (DMFT index), and a change in the distribution of caries experience within the child population in Australia, indicated by increasingly smaller percentages of children accounting for greater proportions of total disease experience. The aim of the present paper was to extend the annual reporting on caries experience in Australia up to and including 1993, and to document the change in the distribution of caries within the child population since 1977. In addition, the data are compared with dental targets for children for the year 2000 in Australia and internationally. Caries data were obtained for the years 1977-1993 for children who were patients at School Dental Services in each State and Territory of Australia. Caries experience was recorded by uncalibrated dentists and dental therapists during routine dental examinations. From 1977-89 data were weighted by State and Territory estimated resident populations. From 1989, the data were stratified according to age, year, and State, and weighted to reflect proportions in the national estimated resident population for each State/age stratum. Between 1977 and 1993 there has been a decline in caries experience for 6 year old children from a diftł of 3.13 to a dmft of 1.90 , and an increase in the per cent with $\mathrm{dmft}=0$ from 33.1 per cent to 53.2 per cent with $\mathrm{dmft}=0$ in 1993 . Over the same time period the DMFT for 12 year olds reduced from 4.79 to 1.10 and the per cent while DMFT $=0$ increased from 10.5 per cent to 53.1 per cent. Projection of the decline in DMFT indicates the dental health target for 12 year old
\end{abstract}

^Australian Institute of Health and Welfare Dental Statistics and Research Unit, University of Adelaide.

†Department of Dental Ecology, University of North Carolina at Chapel Hill, USA.

$\ddagger$ Use of dift - ' $\mathrm{i}$ ’ in this instance represented 'indicated for extraction' which then became dmft after 1989, as the term 'dmft' was in use throughout Europe and America. children of DMFT $=1.0$ by the year 2000 should have been achieved by the end of 1995 .

Key words: Children's dental health, public health dentistry, caries experience.

(Received for publication December 1996. Accepted February 1997.)

\section{Introduction}

The process of developing a health system that is both responsive and accountable to the community requires mechanisms for collecting, analysing and disseminating health information. For example, the determination of need within the community for dental care programmes requires a systematic flow of information between the community and the dental profession. This monitoring process is of particular importance during periods of rapid change in either disease prevalence or the structure and processes of health care delivery. Monitoring provides both an invaluable means for confirming hypotheses based upon clinical observation and for observing differences between comparison groups. Monitoring surveys are also of use for the planning of dental services, where data can be used to provide substance to the process of review, goal setting, and programme selection. ${ }^{1}$

Data on the dental health of Australian school children who are patients of the school dental services have been reported for the period 1977 to $1986,{ }^{2-7}$ where significant outcomes reported included presentations of the secular decline in caries experience as defined by the DMFT index, and a change in the percentage of children with varying levels of caries experience. The oral health of the child population in Australia has also been reported as part of the 1987/88 National Oral Health Survey of Australia, which collected data on Australian residents aged 5 years or more. ${ }^{8}$

Since 1989 dental caries experience with the Australian population of school children has been 
reported annually to States and Territories, and at the national level by the Australian Institute of Health and Welfare Dental Statistics and Research Unit from the Child Dental Health Survey. ${ }^{9}$ Summary caries experience for children aged 6 and 12 years has also been reported in the biennial publication, Australia's Health, ${ }^{10-12}$ and as part of conference proceedings. ${ }^{13-15}$ However, recent information on trends in caries experience over time since 1986 has not been widely available to the Australian dental profession.

The present paper therefore extends the reporting period on trends in dental caries in Australian school children up to and including 1993. In addition, comparisons are made between the data and targets for the dental health of children in Australia for the Year 2000. ${ }^{16}$

The nature of the Child Dental Health Survey also permits the identification of synthetic age cohorts which can be followed over successive years. The presentation of caries experience for different birth cohorts over time permits the examination of ageing, period and cohort effects. A cohort effect is observed in these data by comparing different birth cohorts at the same point in time by, for example, comparing DMFT scores for children across ages five to 12 for the year 1993. An ageing effect is observed by comparing the same birth cohort across sequential points in time by, for example comparing the DMFT scores for a group of children aged 6 years in 1990 with the DMFT scores for the same group aged 7 years in 1991, and so on. A period effect is observed by comparing different birth cohorts at sequential times by, for example, comparing DMFT scores for children aged 12 years in 1977, with children aged 12 years in 1985, and children aged 12 years in 1993.

These different effects can be important where, for example, there is a strong relationship between age and caries experience due to ageing, and concomitant changes in the historical circumstances of prevention and treatment. The interpretation of the relationship needs to consider that only part (if any) of the observed effect may be due to ageingrelated processes, with the remainder of the effect being due to period effects (such as historical changes in exposure patterns to factors such as water fluoridation or fissure sealants).

\section{Aims}

Specifically, the present study was designed to:

- Update the time series on dental caries experience among school children

- Examine the changing distribution of caries experience

- Compare Australian school children's caries experience with targets for the Year 2000.

\section{Data sources and methods}

Data for the present study were drawn from the national Child Dental Health Survey, ${ }^{9}$ and its predecessor, the Australian School Dental Scheme Evaluation Program. ${ }^{7}$ The Australian School Dental Scheme was established in 1973 by the Commonwealth Department of Health in conjunction with the dental services in each State and Territory. The provision of school dental services was accompanied by a survey of children's dental health initiated in 1977, which continued to be coordinated by the Commonwealth Department of Health in Canberra until the end of 1988 when responsibility for the data collection, analysis and reporting was transferred to the Australian Institute of Health and Welfare's Dental Statistics and Research Unit (DSRU). Both sources are collations of data describing dental caries experience of children who are patients of school dental services operated by States and Territories. The distinguishing features of the data sources concern the procedures for selecting children and the index used to measure caries experience of deciduous teeth. When the Evaluation Program was introduced there was complete enumeration of cases for presenting oral health status. However, the expansion of the school dental service resulted in the introduction of sampling to reduce the burden of data collection and analysis. With the transfer of responsibility to DSRU in 1989 there was an accompanying change in most States and Territories to smaller samples reflecting both the population size and the level of disaggregation required in State/Territory specific reporting. Children were sampled on the basis of the date of birth, with for example, the ratio of 1:16 being obtained by selecting children born on the 13th, 30 th and 31 st of any month.

The data presented here were derived from routine dental examinations conducted by uncalibrated dentists and dental therapists working within the school dental service of each State and Territory of Australia. The methodology used for the diagnosis and reporting of caries experience follows those published by the World Health Organization for oral epidemiological studies. ${ }^{17}$ Accordingly, dmft and DMFT indices are used, where dmft is the sum of deciduous teeth which are decayed, missing due to caries, or filled due to caries. The DMFT measure is the corresponding index for permanent teeth. To be recorded as decayed, teeth must have evidence of carious cavitation to the level of dentine.

The unit record data were weighted prior to analysis to reflect age specific estimated resident populations within each State/Territory in Australia. The weights were derived from population Census estimates to adjust for differing sampling fractions and extent of School Dental Service coverage among States/Territories and across age groups. Weighting permits the results to be generalized to the national 


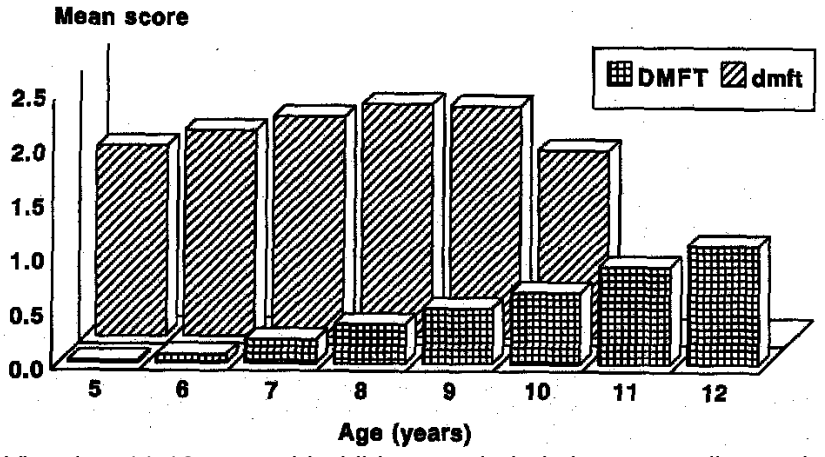

Victorian 11-12 year old children excluded due to small sample, dmft scores plotted only to age 10 .

Fig. 1.-Mean dmft and DMFT scores for children aged 5 to 12 years in Australia in 1993.

population of children receiving care within school dental services.

\section{Results}

Figure 1 presents the caries experience for both deciduous and permanent dentition for school children aged 5 to 12 years for 1993. Deciduous caries experience $(\mathrm{dmft})$ at age 5 years was a mean of 1.80. There was a slight increase across age groups until the peak at 8 years $(\mathrm{dmft}=2.21)$. Deciduous caries experience then declined across older age groups as exfoliation of deciduous teeth, especially deciduous molars which are more likely to have caries experience, becomes more pronounced. As expected, permanent caries experience at age 5 years was negligible. The mean DMFT increased across age groups to a mean of 1.10 by the age of 12 years. However, it is readily apparent that most caries experience of Australian children between the ages of 5 and 12 years was associated with the deciduous rather than the permanent dentition.

Figures 2 and 3 place the data for 1993 in an historical context by presenting the 1993 caries experience data for 6 and 12 year olds as part of a time trend from 1977 to 1993. Caries experience for 6 year old children was 3.13 in 1977 which declined to a dmft of 1.90 by 1993 (Fig. 2). The rate of decline in 6 year old caries experience indicated by $\mathrm{dmft}$ appears to have diminished to virtually zero,

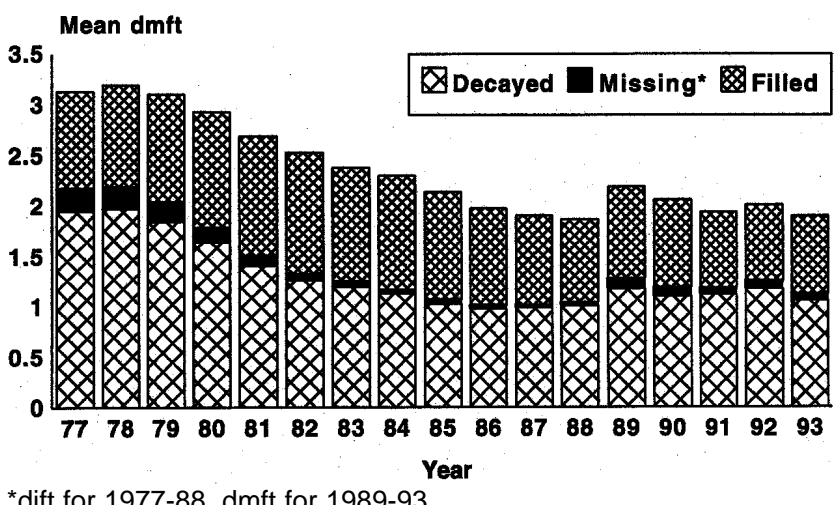

Fig. 2.-Mean dift and dmft components in 6 year old children in Australia from 1977 to 1993.

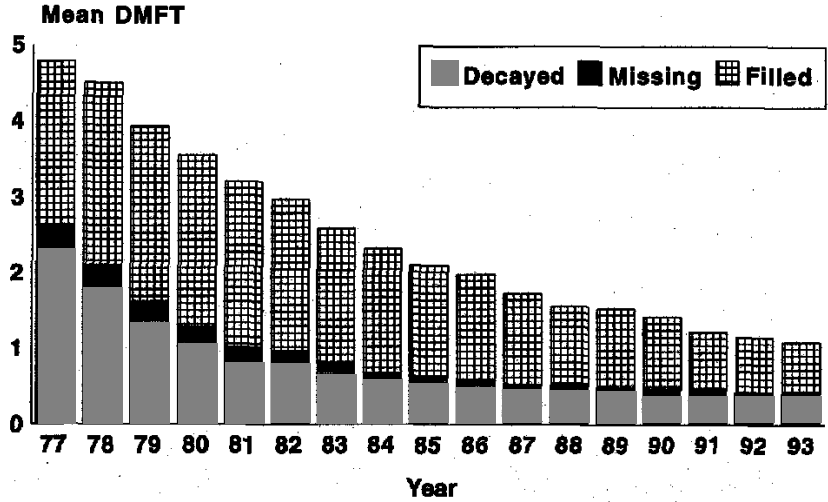

Fig. 3.-Mean DMFT components in 12 year old children in Australia from 1977 to 1993.

with there being no meaningful difference in mean dmft scores between 1986 and 1993. Figure 2 also depicts the components of the $\mathrm{dmft}$ index in 6 year old children over the period 1977 to 1993. Caries experience in deciduous dentition is dominated by untreated decayed teeth, although that domination reduced. The ratio of decayed to filled teeth was 2.05:1 in 1977 and 1.35:1 in 1993. There were low numbers of teeth missing due to caries.

The cessation of systematic decline for deciduous caries experience was not reflected in permanent dentition. Figure 3 illustrates the continued decline of the DMFT index in 12 year olds over the period 1977 to 1993 . The DMFT for 12 year old children was 4.79 in 1977 , which declined to 1.10 by 1993 . A more precise projection of DMFT scores for 12 year olds with regard to this target using a cohort analysis is discussed below.

The decline in mean caries experience, as expressed in DMFT scores, was analysed further by examining the relationship between the components of the DMFT index presented in Fig. 3. In addition to the general decline in aggregate caries experience referred to above, the ratio of decayed to filled teeth has decreased from $1.08: 1$ in 1977 to $0.42: 1$ in 1993.

Further detail of the pattern of change in caries experience is revealed in the distribution of $\mathrm{dmft}$ and

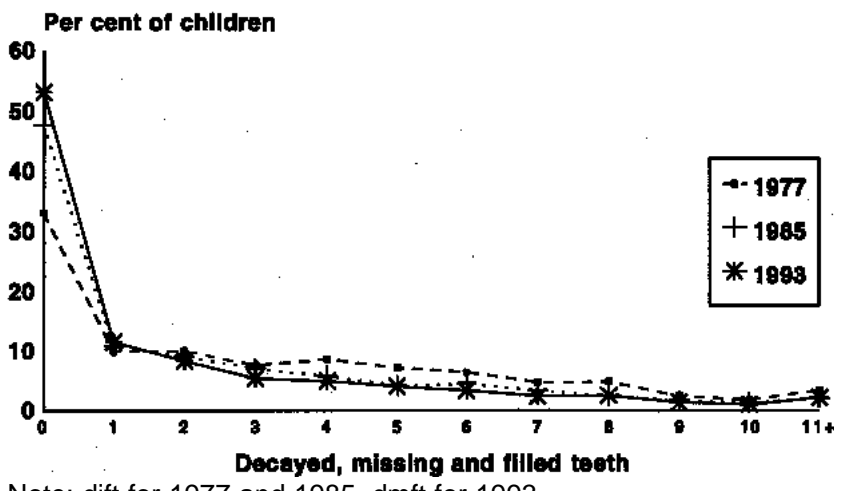
Note: dift for 1977 and 1985, dmft for 1993.

Fig. 4.-Distribution of dmft for 6 year old children in 1977, 1985, and 1993. 


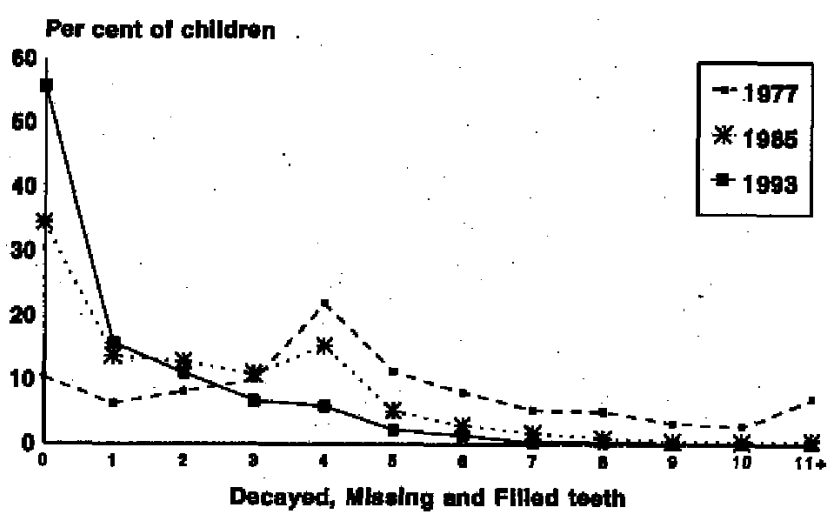

Fig. 5.-Distribution of DMFT for 12 year old children in 1977, 1985 , and 1993.

DMFT. Presented in Fig. 4 are frequency distributions of dift and dmft for 6 year old children for the years 1977, 1985 and 1993. It is apparent that the distributions are very positively skewed, and apart from an increase in the per cent with a zero $\mathrm{dmft}$ from 33.1 per cent in 1977 to 53.2 per cent in 1993 , there has been very little change over time in the shape of the distribution. Moreover, the elevated levels of deciduous caries experience relative to current levels of caries experience in the permanent dentition, and the shape of the disease distribution indicate a substantial burden of disease within the community. For instance, 21 per cent of 6 year old children had a dmft of 4 or more in 1993, and 35 per cent had a dmft of 2 or more.

The pattern of change in the distribution of disease from 1977 to 1993 observed for the deciduous dentition for 6 year olds was dissimilar to that observed in the permanent dentition of 12 year old children. Presented in Fig. 5 are the frequency distributions for DMFT scores of 12 year old children for the years 1977, 1985 and 1993. In 1977, the distribution of 12 year old DMFT was only slightly positively skewed. Only 10.5 per cent of 12 year old children had no experience of dental caries in their permanent teeth, with the most common DMFT outcome by the age of 12 years being a score of four decayed, missing or filled permanent teeth. By 1985, however, the distribution was bimodal, when the most common DMFT score for 12 year old children was zero ( 34.6 per cent),
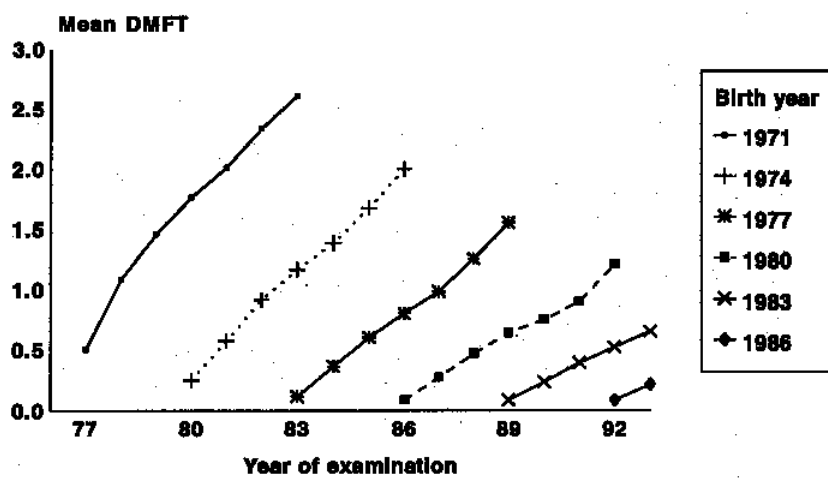

Fig. 6.-DMFT scores for birth cohorts. and a second peak occurred at DMFT of 4 (15.4 per cent). The continuation of the trend for increasing percentages of children to survive to age 12 with no history of clinical caries in their permanent teeth resulted by 1993 in a very positively skewed distribution with no evidence of bimodality, and 55.8 per cent of children at 12 years of age having DMFT scores of zero. A small minority of children had high levels of caries experience in 1993. For instance, while 65.1 per cent of 12 year old children had a DMFT of four or more in 1977, this percentage decreased to 27.7 per cent in 1985 and 11.6 per cent in 1993.

The relationship between ageing, secular change and permanent caries experience is presented in DMFT scores for successive birth cohorts in Fig. 6 . Plotted are lines representing DMFT scores for different birth cohorts by year of examination. For example, the left-most line is for the 1971 birth cohort, where the first point represents the DMFT score during an examination conducted in 1977 when the children were 6 years of age. The second point in the line represents the DMFT score for the cohort in 1978 when the children were aged 7 years, and so on, to the last point when the children were 12 years old in 1983 . Hence, for each birth cohort plotted, the lowest point represents the 6 year old DMFT scores. There are incomplete series for the 1983 and 1986 birth cohorts, as the series finishes in 1993 before these cohorts have reached 12 years of age.

All effects, ageing, period and cohort effects are apparent in the changes in caries experience among Australian children over the period 1977 to 1993. When viewing across cohorts from left to right, there is an apparent reduction in the first point for the birth cohorts, indicating that earlier cohorts had greater caries experience by the age of 6 years. The reduction in the caries experience of a particular age, for instance 6 year olds, in successive birth cohorts represents a period effect. There is also an apparent reduction in the slope of the lines plotted for age cohorts when viewed from left to right, which is consistent with a reduction in caries incidence with ageing for successive cohorts. The DMFT difference between birth cohorts also appears to have diminished over the period reported. For instance, the difference in DMFT between the 1983 birth cohort (aged 6 year olds in 1989) and 1980 birth cohort (aged 9 years old in 1989) is approximately half that between the 1974 birth cohort (aged 6 years old in 1980) and the 1971 birth cohort (aged 9 years old in 1980). This illustrates the cohort effect in the changes observed.

\section{Discussion}

The substantial reduction of caries experience in school children in Australia from 1977 to 1993 represents a significant achievement in dental health 
within Australia. The slight upward turn observed for 1989 is due in part to the inclusion since 1989 of the $\mathbf{m}$ component, as opposed to the $\mathbf{i}$ component used previously in the Evaluation Program of the Australian School Dental Scheme..$^{5-7}$ It is also possible that the altered sampling strategy and additional publicity of the research protocol that accompanied the transfer of responsibility for the survey to the DSRU may have influenced practitioner diagnosis and reporting of deciduous caries experience, resulting in an increase in caries experience.

The 12 year old mean DMFT for 1993 was well below the WHO worldwide target of 3.0 for the Year $2000^{18}$ but greater than the Australian Health for All target for 12 year old children of a DMFT score of 1.0 for the Year 2000. ${ }^{16}$ However, the decline in the mean DMFT score for 12 year olds toward the target for the Year 2000 suggests that this goal will readily be achieved. Indeed, fitting a simple linear model to the cohort that will be 12 years of age in 1995 predicts a DMFT score of 0.96 , which is below the target for the Year 2000 of a DMFT score of 1.0 for 12 year olds.

Examination of the components of the DMFT index indicates that the continuing decline appears now to be largely attributable to reductions in the mean number of filled teeth, as the mean level of untreated decayed teeth has not changed appreciably since 1990. It should be noted that the levels of untreated decayed teeth are low, especially in comparison with the untreated decayed deciduous teeth in six year old children.

The decrease in the ratio of decayed to filled teeth, while the mean number of decayed teeth remains constant, is consistent with the successful targeting of care to children at greater risk of caries experience and the broader adoption of a more conservative philosophy for treating caries in children. It is uncertain from these data how this trend will continue. However, if the decline in the ratio of filled to decayed teeth continues to the point where there is an equal proportion of decayed and filled teeth, and the mean number of decayed teeth remains the same as 1993, the national 12 year old DMFT would be approximately 0.8 . There were negligible average numbers of teeth missing due to caries.

The comparable data for the deciduous dentition presents a less favourable profile of caries experience than for the permanent dentition, with there being relatively stable disease patterns and a comparatively high proportion of the $\mathrm{dmft}$ index being constituted by the untreated decay component of the $\mathrm{dmft}$ index. Despite the improvements in the ratio, young children still enter the school dental services with a high level of untreated decay. It is notable that the $\mathrm{dmft}$ at age 5 was 1.76 teeth, as the great majority of children have their first contact with the school dental service after they commence school at the age of 5 years. This result highlights a priority for both preventive and therapeutic care at an earlier age than routinely provided by school-age based dental care programmes.

The change in the distribution of $\mathrm{dmft}$ has implications for targeting of interventions and services at the minority of children who still have substantial caries experience. The positively skewed distribution of disease with a sizeable proportion of cases clustering around the zero point indicates that caries experience at the age of 6 years is not a normally distributed health outcome within the population. The relatively elevated levels of disease, together with the absence of decline in recent years, and the skewed distribution may indicate the need for both population level strategies to reduce the average level of disease, and further targeted interventions for those children with elevated levels of caries experience. This is in contrast to the DMFT scores for 12 year old children over the same time period where there has been substantial decline in the average level of caries experience, and a dramatic transformation of the distribution of disease. This provides a clear rationale for a minimal interventionist approach with the majority of children with zero DMFT scores, coupled with a programme of targeted care for those children with elevated disease experience.

There are implications also for the future measurement of caries experience, and the provision of services as a result of changes in the observed distribution of caries experience. The use of mean DMFT scores had particular merit when dental caries was ubiquitous. However, the present data lend some support for two alternative measures. The first is the use of a case definition approach to caries, in combination with a severity measure, such as DMFT, for those children with disease. An example of a case definition could include classifying individual caries activity as a DMFT of greater than four, potential caries risk as the presence of two or more non-cavitated enamel lesions, or both high caries activity with further potential risk as indicated by the presence of both non-cavitated lesions in addition to cavitated lesions. A case definition approach represents a reconceptualization of caries experience from being usual within the population to an event that affects a minority of persons. There is practical utility in such a measure, particularly in the development of risk management models for the targeting of care, but also for epidemiological studies where differences in mean scores within study populations are relatively difficult to detect when clinical levels of disease is expressed in only a minority of subjects. The accompanying severity measure could take the form of DMFT scores for those with disease, or the more sensitive surface-specific measure of DMFS which provides a more comprehensive estimate of the extent to which disease and associated treatment have affected tooth tissue. 
The second possibility is to include within the DMFT index a count of both cavitated and noncavitated lesions, the result of which could be a substantial increase in the prevalence of disease under consideration. The consideration of noncavitated lesions is of increasing significance as it can provide additional information on the total burden of caries activity. ${ }^{19}$ This can be used, for example, to compare the ratio of cavitated to non-cavitated lesions as a measure of the effectiveness of caries control in a community.

The existence of the Child Dental Health Survey as a database describing school children's oral health represents the outcome of a continuing commitment within Australia to the study of children's dental health. The observed continued decline in caries experience in children, and the probable achievement of the Year 2000 target of 1.0 for 12 year old DMFT scores by the end of 1995 is a success in caries control programmes within Australia, and allows Australia to compare most favourably with other developed countries. ${ }^{20}$ It is, however, important to emphasize that the programmes presently in place represent an element of control over the risk of caries at a population level, and not the eradication of caries risk for either a population or for any individual. A maintenance of effort is required to ensure that caries experience at the population level does not rise, and an increase in strategic effort is required to address the two problems of continued high deciduous caries experience, and the continued high permanent caries experience in a small percentage of the child population.

\section{Conclusion}

The analyses presented here indicate a dramatic decline in caries experience of children across Australia since the year 1977. It would appear that the target for the Year 2000 of a DMFT score of 1.0 for 12 year old children will be achieved by the end of 1995, but that the decline in 6 year old dmft has ceased at a relatively high level. The changing distribution of caries experience, with the rapid increase in the percentage of children who have DMFT scores of zero at the age of 12 years, provides a rationale for considering additional preventive measures for a minority of children. The modest change over time, and highly skewed distribution of $\mathrm{dmft}$ scores for 6 year old children imply a different strategy to achieve control to that in place for older children. The Child Dental Health Survey, as an example of a national monitoring programme, constitutes a valuable resource for policy development, and monitoring the progress of dental services toward clearly defined targets.

\section{Acknowledgements}

The authors wish to acknowledge the efforts of the school dental service in each State and Territory of Australia in collecting the data used as a basis for the Child Dental Health Survey which forms the basis for the data reported upon here.

\section{References}

1. Spencer AJ. The role of epidemiological surveys in planning dental services: are they a useful tool? Community Dent Health 1985;2:277-83.

2. Carr LM. Dental health of children in Australia 1977-1980. Aust Dent J 1982;27:169-75.

3. Carr LM. Dental health of children in Australia, 1977-1985. Aust Dent J 1988;33:205-11.

4. Carr LM. Frequency distribution of children in Australia according to dental caries experience. Aust Dent J 1985;30:11822 .

5. Commonwealth Department of Health. School dental scheme. Evaluation and statistical data 1977. Canberra: Australian Government Printing Service, 1980.

6. Commonwealth Department of Health. School dental scheme. Evaluation and statistical data 1977-1980. Canberra: Australian Government Printing Service, 1981.

7. Commonwealth Department of Health. Dental health of children in Australia, 1977-86. Canberra: Australian Government Printing Service, 1987.

8. National Oral Health Survey Australia 1987-88. Department of Health, Housing and Community Services. Canberra: Australian Government Printing Service, 1993.

9. Dental Statistics and Research Unit, University of Adelaide. Redesign of the child dental health survey. Adelaide: Dental Statistics and Research Unit, University of Adelaide, 1989.

10. Australian Institute of Health and Welfare. Australia's health 1992: 3rd biennial health report, Australian Institute of Health and Welfare. Canberra: Australian Government Printing Service, 1992.

11. Australian Institute of Health and Welfare. Australia's health 1994: 4th biennial health report, Australian Institute of Health and Welfare. Canberra: Australian Government Printing Service, 1994.

12. Australian Institute of Health and Welfare. Australia's health 1996: 5th biennial health report, Australian Institute of Health and Welfare. Canberra: Australian Government Printing Service, 1996.

13. Davies MJ, Spencer AJ. A review of dental health targets in Australia. Public Health Association of Australia, 25th Annual Conference, Sydney, 29 September to 2 October, 1993. Canberra: Public Health Association of Australia Inc, 1993.

14. Davies MJ, Slade GD, Spencer AJ. Trends in dental caries experience of children in Australia. J Dent Res 1993;72:671.

15. Davies MJ, Spencer AJ. Trends in dental caries experience of children in Australia. Proc Australasian Epidemiological Association, Annual Conference, Cairns, 24 September, 1995:2:14.

16. Health Targets and Implementation (Health for All) Committee. Health for All Australians. Canberra: Australian Government Printing Service, 1988.

17. World Health Organization. Oral health surveys. Basic methods. 3rd edn. Geneva: World Health Organization, 1987.

18. Fédération Dentaire Internationale. Global goals for oral health in the year 2000. Int Dent J 1982;32:74-7.

19. Fejerskov O, Luan W, Manji F. Caries prevalence in Africa and the People's Republic of China. Int Dent J 1994;44:425-33.

20. Spencer AJ, Davies MJ, Slade GS, Brennan D. Caries prevalence in Australasia. Int Dent J 1994;44:415-23.

Address for correspondence/reprints:

M. J. Davies,

Australian Institute of Health and Welfare Dental Statistics and Research Unit, The University of Adelaide, Adelaide, South Australia 5005. 